pathology of the eyes, skin appendages, teeth, central nervous and musculoskeletal systems. The disease manifests in the neonatal period and has four stages: vesicular, verruciform, hyperpigmented and hypopigmented. It occurs mainly in girls, since inheritance has an X-linked dominant character.

A 7-year-old girl, appealed to the department in satisfactory condition, with complaints of mental deficiency, reduced memory, behavior disorders, multiple hyperpigmented spots on the limbs and body. The child after birth had multiple vesicles on an erythematous background on the body, localized linearly, mainly on the flexor surface of the limbs, as well as on the skin of the chest and back. After birth, he was diagnosed with progressive erythema and vesicular rash. In connection with skin manifestations, she was examined for intrauterine infections - pathology wasn't detected. The patient had no family history of skin diseases. Subsequently, the appearance of hyperkeratosis in the form of plaques, warts and lichenoid growths along the Blashko lines was noted in the affected areas of the body. The girl was under the supervision of a pediatrician. During the examination in the Department revealed: mental retardation, hyperpigmented spots of graybrown color with light edges, on the extremities of the rash in the form of 'mud splashes', on the trunk - in the form of a' spiral', ring 'or' marble cake', with the location of pigmentation along the lines of Blashko.

The course of Bloch-Sulzberger syndrome is suspected in a child with clinical manifestations of mental retardation, stages of the skin process with a debut in the newborn period. To confirm the diagnosis, a molecular genetic examination was performed: mutations of the IKBKG gene, which is located on the X-chromosome, were detected.

Typical skin manifestations allow you to clinically suspect the presence of Bloch-Sulzberger syndrome in newborns, to conduct timely molecular genetic diagnostics to make the correct diagnosis. In view, the importance of differential diagnosis in the neonatal period and the rare disease, a multidisciplinary team of neonatologists, pediatricians and neurologists to this disease is necessary.

\section{FIRST EPISODE OF SPORADIC HEMIPLEGIC MIGRAINE IN PEDIATRIC PATIENT- DIAGNOSTIC PHANTOM}

Marija Mirković*, Dubravka Đuraković Fajdetić, Zrinka Ereš Hrvaćanin, Ivana Đaković, Karmen Kondža. general hospital 'Dr.J.Benčević' Slavonski Brod

\subsection{6/archdischild-2021-europaediatrics.406}

The aim of this case report is to describe rare form of migraine in pediatric patient and to point out difficulties of making the right diagnose and classification according to ICHD-III (International Headache Society 2018.)

Case Report A 14-year old girl driven by emergency team to pediatric emergency from school where she had severe unilateral headache with nausea and paresthesia in the right hand and leg about an hour before arrival. At arrival she was confused, unknowing the name of her school and dysphasic. Neurological exam showed dysphasia and right hemiparesis, in the next half an hour she was somnolent, only opening her eyes when shaken and started vomiting.

After two hours (three from the first simptoms) all clinical signs vanished, she was in her full consciousness, ambulatory with no neurological deficits!
-Brain CT, EEG and MRI/MRA were normal, so did Ddimers and coagulogram, we transported her to tertiary level center

Differential Diagnoses 1. cerebrovascular disease- ischemic or hemorrhagic stoke; transient ischemic attack 2. CNS infections/encephalophaty/brain tumor 3. syndrome of transient headache and neurological deficits with cerebrospinal fluid lymphocytosis (HaNDL) 4. migraine

Diagnosis and Classification -Diagnosis is clinical, TIME has major role -It is the diagnosis of exclusion- when we exclude diagnosis from above and time shows that the attack was episodic and reversible, we can than afford ourselves to think about migraine -which type of migraine? According to ICHD -III it does not fulfill criteria for hemiplegic migraine since it takes at least two attacks with motor deficit, so we can only classify this case as 1.5.2: probable migraine with aura.

Conclusion Diagnosis of a first episode of sporadic hemiplegic migraine (severe attack) in a pediatric patient is very challenging for a clinician, especially in a first few hours What makes already dramatic clinical picture even harder to diagnose:

-children and adolescents find it hard to describe their aura symptoms- motor aura is necessary feature for the diagnosis, clinician must establish as clearly as possible whether the patient actually experienced motor weakness as an aura symptom -lack of literature about this cases; no guidelines for the management of acute attacks -signs and symptoms described in all of the other aura types- heterogeneous symptoms Regardless of the new technologies in diagnostic, accessible medical data bases and several headache classifications, some conditions will always remain challenging

\section{VESTIBULAR SCHWANNOMA IN A TEENAGER: CASE REPORT}

Irina Naumova*, AV Pashkov, IV Zelenkova, LS Namazova-Baranova. The Research Institute of Pediatrics and Child Health of the Central Clinical Hospital of the Russian Academy of Sciences of the Ministry of Science and Higher Education of the Russian Federation

\subsection{6/archdischild-2021-europaediatrics.407}

Relevance Vestibular Schwannoma (VS) - is a benign encapsulated tumor, initially arises in the inner auditory canal from the neurolemma of the vestibular nerve with further growth towards the base of the skull. Basically, they are associated with neurofibromatosis type 2 (NF2). In children sporadic cases of VS are uncommon. We report a case of VS in teenager.

Methods A 15-year-old girl with a viral infection was diagnosed with unilateral acute sensorineural hearing loss (SNHL). There was no NF2 family history. General medical examination revealed no ENT-pathology. MRI showed no tumor and vascular malformations in the brain and temporal region. After the standard course of dexamethasone effect was not obtained. SNHL progressed over the next 9 months. Evaluation of advanced hearing tests showed: tympanometry (probe-ton 226 $\mathrm{Hz}$ ) demonstrated Type A bilateral, TEOAE were documented as 'Pass' bilateral, pure tone audiometry determined profound hearing loss on the right, Auditory Brainstem Response (ABR) was defined wave $\mathrm{V}$ latency on the right, ABR thresholds were detected with intensity stimulus level $85 \mathrm{~dB}$ SPL on the right and $30 \mathrm{~dB}$ SPL on the left. At the series X-ray CT images of temporal region were determined inner auditory canal deformation and extension. A second MRI scan revealed a bulky tumor coming from the inner auditory canal. The 